\title{
The sense of agency - a phenomenological consequence of enacting sensorimotor schemes
}

\author{
Thomas Buhrmann ${ }^{1}$ Ezequiel Di Paolo ${ }^{2,1,3}$
}

Published online: 12 November 2015

(C) The Author(s) 2015. This article is published with open access at Springerlink.com

\begin{abstract}
The sensorimotor approach to perception addresses various aspects of perceptual experience, but not the subjectivity of intentional action. Conversely, the problem that current accounts of the sense of agency deal with is primarily one of subjectivity. But the proposed models, based on internal signal comparisons, arguably fail to make the transition from subpersonal computations to personal experience. In this paper we suggest an alternative direction towards explaining the sense of agency by braiding three theoretical strands: a world-involving, dynamical interpretation of the sensorimotor approach, an enactive description of sensorimotor agency as contrasted with organic agency in general, and a dynamical theory of equilibration within and between sensorimotor schemes. On this new account, the sense of oneself as the author of one's own actions corresponds to what we experience during the ongoing adventure of establishing, losing, and re-establishing meaningful interactions with the world. The meaningful relation between agent and world is given by the precarious constitution of sensorimotor agency as a self-asserting network of schemes and dispositions. Acts are owned as they adaptively assert the constitution of the agent. Thus, awareness for different aspects of agency experience, such as the initiation of action, the effort exerted in controlling it, or the achievement of the desired effect, can be accounted for by processes involved in maintaining the sensorimotor organization that enables these interactions with the world. We discuss these processes in detail from a non-representational, dynamical perspective and show how they cohere with the personal experience of agency.
\end{abstract}

Thomas Buhrmann

thomas.buehrmann@gmail.com

1 IAS-Research Center for Life, Mind, and Society, Department of Logic and Philosophy of Science, University of the Basque Country, UPV/EHU, San Sebastián, Spain

2 Ikerbasque, Basque Foundation for Science, Bizkaia, Spain

3 Centre for Computational Neuroscience and Robotics, Department of Informatics, University of Sussex, Brighton, UK 
Keywords Enactive cognitive science -Agency - Sense of agency · Sensorimotor contingencies $\cdot$ Equilibration $\cdot$ Metastability

\section{Introduction}

Embodied accounts of motor control and perceptual experience fall within one of two categories according to the explanatory role they give to the agent's body and to the world. In-the-head approaches put the emphasis on computational processes occurring in the agent's brain that instantiate internal models of body and world. Such models can be affected by environmental events, sensorimotor regularities, and body dynamics in a limited way: either in the form of informational inputs or via the 'formatting' of internal representations. In-the-head explanations are subpersonal and internalist. Worldinvolving approaches, in contrast, see brain, body, and world as part of an entangled relational network of processes in which both neural, non-neural, and non-biological elements can play strong causal and constitutive roles - not just informational or developmental ones - in action and perception. The explanatory strategy in such cases require establishing links between the personal-level relation between agent and environment as well as the dynamic coupling of subpersonal processes in the agent and in the environment.

The most popular accounts of the sense of agency, the experience of being the author and initiator of our own actions, are in-the-head approaches. They involve comparisons between intended and actual states of the body and the world, as if experiencing oneself to be the agent of an action were first and foremost a question of verifying whether these states match or not. Our objective in this paper is to introduce a world-involving alternative account based on O'Regan and Noë's (2001) sensorimotor (SM) approach to perception; more specifically based on recent formalizations of this proposal (Buhrmann et al. 2013; Di Paolo et al. 2014). This alternative does not see the sense of agency as just an epistemological problem, but rather assumes that it is an intrinsic aspect of how sensorimotor schemes are organised and enacted in the world.

A sense of the bodily self as an agent, in this view, corresponds to what we experience during the ongoing adventure of establishing, losing, and reestablishing meaningful relations between ourselves and the world. This is what an enactive account of the sense of agency should endeavour to explain by articulating in operational terms what these meaningful relations consists of, as well as what it means to establish them or to lose them. To do so we need to elaborate further links between the sensorimotor approach and enactive ideas (Di Paolo and Thompson 2014; McGann et al. 2013; Thompson 2007; Varela et al. 1991), in particular by extending the account of minimal agency provided by Barandiaran et al. (2009) to the sensorimotor realm. The latter step enables us to address an issue unsolved by the SM approach thus far, namely the problem of why the enactment of sensorimotor contingencies should be accompanied by any subjective perspective at all, and what sorts of systems are able to enjoy subjective experiences.

We propose that the various aspects of the phenomenology of the sense of agency relate to both the intrinsic and the relational (meta)stability of the action/perception 
schemes that together constitute the sensorimotor level of agency. These intrinsic and relational aspects always involve the world in some non-trivial sense and do not require internal comparison between neural signals as the epistemic signature of a controlled act. Instead, the enacted schemes "belong" to the agent to the extent that they assert her agency in the first place. This is manifested in different forms: as feelings of action initiation, of action control, of effort and control exertion by the various ways and degrees in which an enacted scheme is met with, and surpasses (or not), obstacles and resistance both internally within a given act and relationally between acts.

Our enactive account of the sense of agency is situated at the meso-level between neurodynamics and personal experience. In developing it, we follow the general research direction of neurophenomenology (Varela 1995, 1999; see also Gallagher 1997; Lutz and Thompson 2003) according to which phenomenological descriptions of lived experience and naturalistic explanations are contrasted in order to uncover their mutual constraints and compatibilities. As a matter of convenience, we will often talk about "explanations" or "accounts" of the sense of agency. The use of this terminology should not be understood as an attempt to reduce experiential aspects to naturalistic processes, but as an effort to provide a neurophenomenologically coherent story. We also follow Beaton (2013) in acknowledging that, pace the label, neurophenomenology need not be restricted to neural processes, but may involve complex linkages between brain, body, and world. In short, neurophenomenology can be world-involving.

In the following section we review the phenomenology of the sense of agency as well as key findings and concepts concerning its normal and pathological aspects. Section 3 describes and criticises the comparator model, currently the most widespread explanation of the sense of agency. Before detailing our alternative theory, we provide the necessary background for its key elements in section 4 . This requires reviewing some recent theoretical developments in the sensorimotor approach, introducing the enactive notion of sensorimotor agency, and summarizing our dynamical theory of equilibration within and between sensorimotor schemes. Based on this, we develop in section 5 a new proposal for a world-involving account of the sense of agency.

\section{The phenomenology of agency}

In everyday life, when I engage in intentional actions, these are usually accompanied by an experience that I am their author or initiator, in other words, by an awareness that the actions are mine, and that I have caused them. Upon reflection, and based on empirical data which we summarize below, this sense of agency is not a unique and unified sense. Rather, one can distinguish different levels of action awareness, and various aspects of one's agency at which this awareness can be directed.

We should note, before continuing, that the phenomenology of agency is itself a hotly debated topic. Not only do researchers disagree on its level of explicitness, or the mechanisms giving rise to it, some also flatly deny the existence of any distinct experience beyond the level of conscious intentions and expectations in one's actions. We will come back to this in the discussion. For now, we will take the following account of agency experience as a starting point and will show how an enactive approach to sensorimotor agency may shed light on its origin. 


\subsection{Pre-reflective and reflective sense of agency}

At a general level we can distinguish between a pre-reflective and a reflective selfawareness in action (Gallagher 2007, 2012). The former, also referred to as the feeling of agency (Synofzik et al. 2007, 2008), is the experience of agency that accompanies my actions when I'm immersed in my activity, without paying particular attention to or consciously reflecting on the details of what I'm doing at the moment or why. At this level, my agency is not given to me explicitly as an object of experience, it is rather implicit in the unperturbed flow of my action and the egocentric perspective underlying it. It is the basic, diffuse feeling that it is I who is carrying out an activity, but the I here is implicit in the non-transitive experience of myself as the locus of agency. It is phenomenologically recessive in the sense that in normal circumstances I am primarily aware of what I'm doing, rather than the fact that it is I who is doing it. Often we become consciously aware only of the absence of the feeling of agency when being interrupted while immersed in a task, or when unexpectedly failing in some way.

We can also experience ourselves reflectively as agents when taking an introspective stance that is detached from our ongoing activity. For example, when deliberating and planning actions we are about to take, or when explicitly monitoring the success of our actions, we may judge ourselves to be responsible when the actions are consistent with our personal beliefs, or when the task results in the achievement of a goal I have set to myself. This sense of agency is usually conceived as a higher-order, conceptual attribution, and a transitive experience of myself as object, i.e. as he who is acting.

The two forms of agency experience are separable. This is demonstrated, for example, in anarchic hand syndrome (Marcel 2003; Marchetti and Della Sala 1998; Pacherie 2007a), one of several symptoms involving a disturbed sense of agency. Patients with certain types of neurological damage, who with their contra-lesional hand perform complex, well-formed and intentional (goal-oriented) actions in response to environmental cues, such as operating a doorknob or scribbling with a pencil (Frith 2005), report that these actions are independent of their will, i.e. as if they occurred involuntarily. Of particular interest is the case of a man who disowned the anarchic actions that his hand was performing, reporting that he was not doing them, yet maintained "of course I know that I am doing it. It just doesn't feel like me" (Marcel 2003, p. 79, emphasis ours). Based on such observations, Marcel argues that the person with anarchic hand "is often clear that their experience of the action as disowned is a "seeming"" (ibid.), which illustrates how the first-order feeling of agency, and the second-order judgment of agency are indeed separate processes, and that they may come apart, at least in pathological cases.

\subsection{Aspects of pre-reflective agency experience}

Before elaborating on the different aspects of actions that one can be aware of, and which may contribute to the overall feeling of agency, we should separate the sense of agency as described above from the sense of ownership (Gallagher 2000; Synofzik et al. 2008). The latter is the pre-reflective experience that it is me, i.e. my body, that is moving, or more generally, that a given body part belongs to me. In everyday voluntary activity these two aspects contribute to a unified, minimal self-awareness for action. That they are nevertheless distinct phenomenological aspects is revealed, for example, 
in involuntary movements. In such cases, e.g. when being pushed, I may lack a sense of having initiated the movement, or of controlling it, yet I may still have the sense that it is me who is moving.

\subsubsection{Intentional and movement-related aspects}

Under normal conditions our own agency tends to present itself as a unified experience. I may or may not feel as if I am the author of my actions, but usually this feeling does not present itself in varying shades or qualities, in the same manner, say, that the visual appearance of a surface may differ in terms of perceived light intensity, colour, texture, and so on. However, experimental manipulation and phenomenological analysis reveal that there are multiple different aspects that can contribute to the overall feeling of agency.

Gallagher (2007, 2012), for example, distinguishes intentional aspects involved in the sense of agency from those related to the initiation of movement. The distinction is motivated by the observation that in the case of involuntary movements I have a sense of ownership only (but not agency), which is based on afferent sensory feedback (e.g. proprioceptive and kinaesthetic). What is different in the sense of agency during voluntary movements, is the presence of efferent signals sent to the motor systems. A series of experiments reported by Tsakiris and Haggard (2005) highlight the role of efferent signals in the sense of agency during movement initiation. Using an independent manipulation of efferent and afferent signals (e.g. arm movements executed voluntarily vs. involuntarily), the authors show that the presence of efferent motor signals is important, for example, to attenuate the sensory consequence of selfgenerated movements (even when the consequences cannot in principle be predicted), or to distinguish between one's own hand passively moving, and that of an experimenter performing the same movement.

Another element of agency experience that is related primarily to bodily movement rather than the intentional aspect of action, is the sense of being in control of ongoing movements, as distinct from the sense of having initiated them. De Vignemont and Fourneret (2004) describe pathological cases where the two aspects are differentially affected. For instance, patients with anosognosia for plegia (unawareness or denial of paralysis) may believe that they have raised their hand, even though they suffer from a condition preventing them from voluntarily initiating any movement with the affected limb., i.e. their sense of initiation is disrupted. In contrast, deafferented patients who do not receive any tactile or proprioceptive feedback have no sense (other than visual) of their own movement or body position, yet they know perfectly well whether or not they are moving.

Intentionality enters into the phenomenology of agency awareness in two ways. The first is the feeling that not only have I initiated a certain action, but that my initiating it is in accordance with my intention to do so. Patients with schizophrenic delusions of control, for example, seem to have a perturbed sense of the latter kind. The symptoms in this condition are in some respects similar to anarchic hand, in that patients will report not to be the agent of movements they are carrying out. But the two conditions are different in crucial ways. In anarchic hand, patients often carry out actions that are against their conscious will. They will recognise this and try to stop them. Patients with delusions of control, in contrast, often continue to make the movements they intended, 
such as when following the instructions of an experimenter. They moreover seem to have awareness of the intentions underlying their movements, since they do not try to stop or correct them. Yet, they insist that their movements are being controlled by external forces, reporting to believe, for example, somebody has implanted a computer in their brain that is making them move, or feeling as if being steered around (Frith et al. 2000b). These patients are aware of their intentions, and of the movement as it occurs (as well as having a sense of ownership), but not of having initiated it. Patients seem to experience a feeling of somebody anticipating their actions (reading their intentions), and initiating them without their intervention (ibid.). Delusions of control thus illustrate how the willing of an action and its initiation are separable aspects that can be experienced independently.

A second intentional aspect of agency awareness relates to the desired outcome of an action. An inherent property of intentional actions is that they are directed at achieving a meaningful effect. The extent to which I am successful in achieving this effect can enter as another element in my agency experience. Since the success of my action usually requires certain outcomes in the world, it is not surprising that this experience depends to a greater extent on the ability to monitor distal action effects, rather than internal processes and signals. As a result, action-effect awareness seems to be easily manipulated and can be falsely induced, even in non-pathological situations. In experiments by Wegner and Wheatley (1999), for example, subjects report to have authored an action even if the corresponding movement had in fact been produced by another participant sharing the control device, and simply because the subject had been exposed to a movement-congruent word just prior to the movement. This sense of agency relies on the perceived contingency between mental states and action effects. As long as the action outcome is consistent with one's intention, follows the intention within a certain time window, and there is no other conspicuous cause, then the action can be experienced as intended and effectuated by oneself.

What is the relationship between the different aspects of pre-reflective action awareness? It is clear that under normal circumstances we do not experience these aspects in isolation. They rather seem to enter into a unified, qualitative and diffuse sense that it is I who is in control of my actions. Moreover, as Marcel (2003) argues, few if any of the individual aspects seem to be necessary for the experience of agency. For example, neither a sense of ownership based on proprioceptive awareness, nor (conscious) awareness of the reasons for our actions seem to be required in order to feel that we are performing intentional actions (e.g. anarchic hand). At the same time, most of the different aspects may be sufficient on their own to create a sense of agency. For example, merely being aware of one's intention to move (or of one's attempt to initiate a motor response), can be sufficient to feel that one is engaged in action, as is the case in anosognosia for plegia. Like Marcel, Gallagher (2007, 2012) as well as Synofzik et al. $(2007,2008)$ therefore defend a view of the sense of agency as involving a complex integration of afferent, efferent, and intentional feedback.

\section{The comparator model}

In-the-head approaches, which emphasize computational processes in the brain that produce and consume representations, such as facts about my intentions and the actions 
I perform, seem to dominate the current landscape of theorizing about the sense of agency. A common model is based on the concept of efference copy, introduced in the early 19th century (Steinbuch, 1811/2012). This concept refers to the idea that appropriately transformed copies of motor commands can be used to modulate afferent signals, in order, for example, to cancel out sensory signals resulting from selfgenerated movements (re-afference; see von Holst and Mittelstaedt 1950). An updated version of this proposal (Fig. 1) postulates detailed internal models that predict the proximal and distal sensory consequences of an action, and which can serve in comparisons with either actual sensory feedback or the desired state of the agent (for predictive in-the-head models of cognition in general also see Wolpert et al. 2003; Knill and Pouget 2004; Grush 2004; or Seth 2014).

Frith et al. (2000a,b; see also Synofzik et al. 2008), for example, propose that a match between predicted and desired state underlies a sense of being in control (comparator $\mathrm{C}_{1}$ in Fig. 1). In anarchic hand, for instance, the patient's movements are triggered directly by environmental affordances, rather than what the patient holds to be her intentions. Predictions made on the basis of involuntarily executed motor commands would thus differ from the desired state as derived from the subject's goals. The resulting discrepancy could hence be experienced by the subject as a lack of control over her own actions. Similarly, in patients with delusions of control, the predicted state is supposed to be unavailable for monitoring. Again, a false discrepancy is thus generated at the comparator, even though awareness of intention and action execution is normal. The absence of an awareness of having initiated actions, so the authors suggest, is tied to an absence of predicted action consequences prior to movement.

The comparison of predicted and actual (estimated) state, in contrast, allows for the self-attribution of sensory events (comparator $\mathrm{C}_{2}$ in Fig. 1). In this scheme, if the actual state matches one's prediction, i.e. if events that have occurred can be "explained" completely by what one has intended, then such events are attributed to one's own

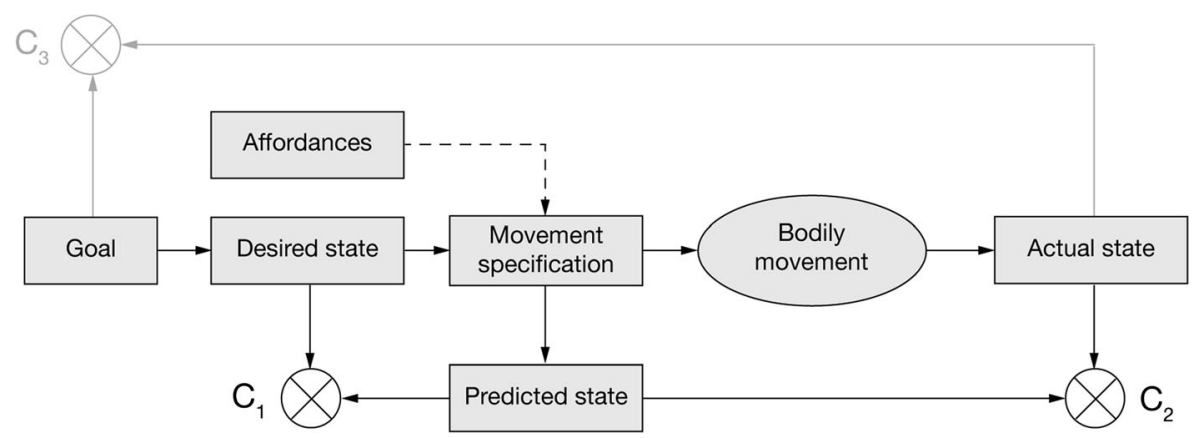

Fig. 1 The computational motor control hypothesis of action awareness. A person's intentions or goals are first translated into a desired state (bodily or world). Using an internal inverse model, motor commands are computed that are appropriate for achieving this desired state (motor specification). The resulting bodily movement leads to sensory feedback (proximal and distal), from which the actual state of the body and world are estimated. The result of the comparison between predicted and desired state $\left(\mathrm{C}_{1}\right)$ is supposed to underlie the sense of control, while the comparison between actual and predicted state $\left(\mathrm{C}_{2}\right)$ allows for self-attribution of sensory events. Awareness of action-effects, i.e. the sense of achieving what was intended, involves the comparison between one's goals and the state of the world $\left(\mathrm{C}_{3}\right)$. In patients with anarchic hand or utilization behaviour, movements are triggered directly by environmental affordances, which override the subject's conscious intentions 
agency. The absence of any discrepancies at the comparator manifests itself in the attentive recessiveness of the feeling of agency. By contrast, if actual sensory feedback is incongruent with sensory predictions, external causation of the sensory stimuli can be inferred (ex-afference). According to Frith et al. (2000a), in patients with anosognosia, motor commands normally initiating actions do not lead to any movement (due to paralysis), while predictions are still made based on their motor intention. The disorder, in this case, results not from the corresponding discrepancy between predicted and actual state, but from the patients' inability to register this discrepancy.

According to the comparator hypothesis, the pre-reflective feeling of agency is thus a diffuse sense that results from the matching flow of predictions and sensory feedback, which occurs at the subpersonal level and is phenomenologically recessive, in the sense that one normally becomes aware only of disruptions of the process, i.e. when one's anticipations are not met (Synofzik et al. 2007).

Despite its appeal, in proposing a simple computational mechanism underlying sensorimotor self- awareness, which moreover seems to fit naturally into the growing ecology of predictive brain theories (Friston 2010; Clark 2013; Seth 2014), the comparator model is likely neither sufficient nor necessary to explain the feeling and judgement of agency. Synofzik et al. (2007) present a range of arguments to this effect. Firstly, subjects can attribute the same comparator mismatch in some cases to themselves and in others to the world. In other words, any potential mismatch itself has to be registered and appropriately categorized by another process different from the comparator. Also, in order to learn the required internal models, i.e. to learn the effect of its own movements, the system already has to somehow know which of its movements are caused by itself and which are not. Based on cases of pathological loss of action awareness, as well as neuroanatomical lesions in areas supposed to be involved in the comparator, the authors reach the conclusion that a much less specific congruence between efferent and afferent signals in general (e.g. between an action intention and a distal sensory effect, see comparator $\mathrm{C}_{3}$ in Fig. 1), alone or in combination with certain intermodal congruencies, suffices to explain the feeling of agency. This idea is supported, for example, by patients who feel agency for phantom limbs (Ramachandran and Rogers-Ramachandran 1996), i.e. in the absence of proprioceptive feedback as well as efference copy. Similarly, in the "helping hands" pantomime task, passive subjects experience high degrees of agency for movements that are in fact performed by another agent, when only the other agent's hands appears in the place where the subject's hands would normally appear (Wegner and Sparrow 2004), i.e. when there is most plausibly no efference copy and no comparator involved.

Synofzik et al. conclude that it is not a specific, unique and accurate prediction that underlies different forms of action awareness. Rather, all kinds of action-related perceptual and motor information, like efference copies, sensory feedback modalities and their congruence, are combined in a multifactorial weighting process at different levels of cognitive processing, where the importance of the different authorship cues may vary with task, context, and person.

Apart from the question of whether comparators are necessary or sufficient for different aspects of action awareness, another problem the approach faces concerns the transition from subpersonal computations to an account of personal-level agency experiences. Note that it cannot be simply the presence of certain computations and resulting measures of mismatch, which constitutes the subjective sense of agency. If 
this was the case, then one would have to grant agency awareness also to thermostats and simple robotic devices. Computations such as those involved in the comparator model may hence play at most an enabling role in the sense of agency. As Synofzik et al. (2007) have pointed out, whether the results of such computations contribute to the experience of agency or not, must be decided by processes different from the comparator itself. The sense of agency, on this view, is thus not in fact an "intrinsic property of the action processing itself" (ibid. p. 4; also see Christoff et al. 2011, for a similar claim).

The comparator model on its own does not offer an answer as to the experiential nature of agency awareness. To do that, it needs to go a step further, and explain how it is that we have these first-person, subjective experiences at all, accompanying (and conditioned by) the proposed subpersonal computations. Although this aspect is less well developed, the proposal is that the result of the comparator enters into the creation of internal representations of "mineness" that become available to other cognitive subsystems (Synofzik et al. 2008). In the case of the non-reflective feeling of agency, these representations are supposed to be non-conceptual, yet abstracted from ongoing action instances. They classify actions as caused by the self or not, but without explicit attribution. They are not compositional (they are not composed of parts representing the self and the fact of it being the cause of action), and have no object-property structure. The reflective judgement of agency, in turn, is supposed to arise from further processing of these primary representations, now involving conceptual capacities and beliefs, which results in an explicit, propositional and compositional kind of representation of actions as one's own (ibid, pp. 415).

Even if a meaningful interpretation could be given to the idea of non-conceptual representations, it is clear that whether one believes the comparator approach to be fruitful hinges on one's acceptance or not of experiences as fundamentally representational in nature (Metzinger 2000; Dretske 2003). In light of the pragmatic turn in cognitive science (Varela et al. 1991; O'Regan and Noë 2001; Noë 2004; Engel et al. 2013), such doubts may at least warrant the exploration of less representation-centric alternatives. We do not claim, of course, that all non-representational approaches to action are necessarily compatible with the phenomenology of prereflective agency described in section 2. But we believe the following account not only matches this phenomenology well but may also shed some light on its origin.

\section{Enactive sensorimotor theory}

As we have argued above, cognitivist models aim at a view of action awareness in which the experience of agency arises as an intrinsic aspect of action itself (at least in the case of the pre-reflective feeling of agency). It is arguable whether this attempt could be successful, given that it relies on the explicit construction of internal representations from brain-side computations that may or may not be required for the sense of agency. We propose here an alternative that synthesizes three different theoretical developments, each of which views the cognitive agent essentially as an integrated ecology of sensorimotor skills. The starting point is a dynamic interpretation of the sensorimotor approach, which points the way towards a world-involving and nonrepresentational account of experience. This is enriched by the enactive notion of 
minimal agency, which we argue is required to explain how subjectivity can arise at the sensorimotor level. Lastly, a dynamical reinterpretation of Piaget's theory of equilibration will allow us to account for the different aspects of the sense of agency.

\subsection{Dynamical, non-representational account of SMCs}

We agree with the premise that the co-occurrence of actions and their typical sensory consequences (i.e. sensorimotor contingencies), as well as a sensitivity to non-typical consequences, are necessary preconditions for the sense of agency, as they may enable a momentary and implicit distinction between self and environment. However, this idea can be developed in a non-dualistic and non-representational manner, in which agency experience is truly intrinsic to the performance of actions themselves as they form part of self-asserting sensorimotor structures and relations, rather than derived through verifications and inferences using pre-given criteria as to what does and does not belong to the self.

According to the sensorimotor approach (O’Regan and Noë 2001; Noë 2004), perceptual experience, such as seeing an object, does not derive from internal representations in the brain, but is constituted by the skilful use of the regularities governing active exploration of the world. More specifically, perceiving consists in the exercise of practical mastery of the laws of sensorimotor contingencies (SMCs), i.e. of the lawful regularities in the sensorimotor flow that govern intentional interaction with the environment. Both the "content" and form of experience, i.e. what is perceived and how, is constituted by the embodied know-how of the relevant SMCs put into practice. The experiences associated with the various sensory modalities, or with distinct aspects of the environment (e.g. colours or sounds) differ, because there are different sensorimotor regularities involved in, say, seeing and hearing.

Our proposal is to extend the sensorimotor approach to the experience of oneself as an agent. On this account, the sense of agency is not something derived from internal representations of our own action-related processes. Rather, it is essentially another dimension of our relation with the world, and derives from the ways in which we establish, lose, and re-establish meaningful interactions between ourselves and our environment. In the same way that certain regularities in the sensorimotor flow associated with the enactment of SMCs determine the qualitative character of perceptual experience, certain features of the processes underlying the mastery and deployment of SMCs determine the character of agency experience.

We should note that when we talk about SMCs here, we refer to a specific dynamical interpretation of the concept (Buhrmann et al. 2013). This interpretation allows for the distinction between different kinds of SMCs, two of which are of particular relevance in the current context: sensorimotor coordinations and strategies. Sensorimotor coordinations describe particular sensorimotor patterns that are reliably used in performing a task. These can be cycles or transients in sensorimotor space and depend on an agent's environment, body, inner activity, and the task-related context. Sensorimotor strategies or schemes are organizations of several sensorimotor coordinations that the agent deploys to achieve a given task and which are subject to some normative framework (for instance, considerations of efficiency).

Crucial for the development of our proposal is that individual SM structures do not exist in isolation. Rather, they are integrated in a complex network of interdependence. 
For example, my going to work may require that I first pick up my keys in the kitchen, lock the door, enter the car, drive to the office, and so on. At the same time, the sensorimotor scheme of picking up my keys is required in other contexts, such as going to buy milk from the corner shop. Everyday skilful coping thus relies on the deployment of a network of interlocking sensorimotor schemes.

What do we mean by deployment of SMCs? How exactly does a subject engage a particular SM scheme in his repertoire? For example, sitting at my desk, what determines whether I reach for the mouse to open my inbox in response to an incoming email, or rather for the glass of water to quench my thirst? Here we outline a plausible answer. Though constitutively involving the environment to close the sensorimotor loop, the use of SMCs is supported on the brain side by metastable neurodynamic structures in conjunction with metastable states in other non-neural systems in the body (see e.g., Tognoli and Kelso 2014). Like, for example, dynamic fields (Erlhagen and Schöner 2002; Schöner and Dineva 2007) such neurodynamical patterns may be characterised by a continuous distribution of activation. One may imagine the neural assemblies supporting different schemes as exhibiting localized peaks in this distribution, for instance (say, one for mouse control and one for grabbing the glass). If the activation of any such peak is large enough, the corresponding scheme is engaged, i.e. the neuro-dynamics engages the motor system. If the environmental conditions are supportive, and the motor system is in a compatible state (e.g., not prevented from moving), then the corresponding scheme is enacted by the agent.

Different sources of activation may prime individual SM schemes by raising their excitability, such as internal signals related to volition and readiness (or my thirst), as well as environmental affordances (the presence of the glass or computer mouse). The activation-threshold model is just one possible way of conceptualising this relation between different metastable neural and bodily states, rich in potentialities, which according to the circumstances can get differentially actualised in the initiation of a particular action. Other models also fit this account in terms of critical states (see e.g., Kostrubiec et al. 2012; Wallot and Van Orden 2012). In a general sense, the enactment of a particular SM scheme depends in normal circumstances on a resonance between external and internal conditions related to the agent's desires and needs. Bodily states, history, dispositions and external factors may resonate with the water-drinking scheme more intensely or sooner than with the email-reading scheme, and it is this resonance between agent and world that breaks a symmetry in the critical state and tips the balance. As the agent is involved in regulating its coupling with the environment, an activity that in turn affects its own states, it is conceivable that some agents may be able to influence the process of selection of SM schemes, as a form of higher order regulation. This may take place through environmental mediation (e.g., the arrangement of a work-environment to encourage "good habits"). But it may also involve internal "gestures" that differentially amplify specific neurodynamic patterns. This is clearly the case in agents possessing linguistic abilities and capable of using them for their own self-control (Cuffari et al. 2015). On lower levels this may include the kind of volitional, readiness or appetitive signals mentioned above. In both these cases-via alterations in the world, or via internal gestures-the whole agent is involved in the process by encouraging certain SM schemes to resonate with the situation while others are avoided or suppressed (see also Fuchs 2011). 


\subsection{Sensorimotor agency}

The idea as sketched so far is not yet sufficient to address the weaknesses we have identified in the comparator model. This is because the SM approach explains the origin of differences between sensory modalities or instances of perceptual experiences, but as Thompson (2005) has noted, it does not address the question of why we have experiences at all, i.e., the problem of creature consciousness. In the same way that a self-guiding missile, despite claims to the contrary (O'Regan and Noë 2001), does not have genuine mastery of airplane-tracking, and hence no genuine perceptual experience of "seeing" airplanes, the sensorimotor account alone is insufficient to describe the kinds of systems that can experience themselves as agents. What is missing, we argue, is an explicit notion of sensorimotor agency, i.e. a naturalized concept of a sensorimotor-based subject, with an intrinsically defined perspective, able to engage in intentional actions subserving her desires and norms. It is necessary but not enough to claim, as Hutto and Myin (2013) do, that "the phenomenological character of experiences must, ultimately, be understood by appealing to interactions between experiencers and aspects of their environment" ( $p$. 176-77). Even if it was true that by defining phenomenological experience, such as of oneself as an agent, exactly as a relation between the acting subject and its environment, one may thus avoid having to explain the otherwise mysterious transition from mere physical happenings to an agent capable of experiential phenomena, this would only reframe the problem as the question of what an "experiencer" is in the first place, i.e. what is it about such entities that they can have experiences. We suggest that an enactive framing of the sensorimotor approach can resolve this issue.

We propose that the origin of a first-person perspective, a prerequisite for talking of a system enjoying experiences, is the emergence of sensorimotor agency. By this we mean an entity whose constitution implies a unique and intrinsic perspective on the world; or rather, an entity that, through its self-constitution, brings forth its own domain of relevant interactions, or Umwelt in von Uexküll's (1934) terms. This is most easily understood in analogy to biological agency. From an enactive perspective, the simplest living organisms already exhibit a form of agency. Unicellular organisms, for example, are complex networks of precarious, co-dependent chemical reactions, in which the activity of the whole network is necessary to prevent the component processes from running down. These cells are agents in the sense that they can regulate interactions with their environment in a way that support their continued existence (e.g. by regulating osmotic pressure). Otherwise neutral external affairs thus gain a valenced status with respect to the cell. What is good or bad for it is not arbitrarily defined by an external observer, but is intrinsically determined by its processes of self-constitution. Thus, unicellular organisms can be said to enjoy a certain subjectivity or perspective in the sense of "sentience, the feeling of being alive and exercising effort in movement" (Thompson 2007, p. 161, see also Jonas 1966; Sheets-Johnstone 1999; Margulis 2001).

Barandiaran et al. (2009) have synthesized this idea into an operational definition of minimal agency that is captured in three individually necessary and jointly sufficient conditions. The first is individuality, the requirement that through its own activity the system distinguishes itself from its surroundings in a non-arbitrary fashion. Organizational closure, a relation between component processes in which each precarious process in the system is enabled by and in turn enables at least one other process, is one way a system may realize its ongoing individuation. 
The second requirement for agency is interactional asymmetry: the system's ability not merely to passively suffer exchanges, but to actively modulate its interaction with the environment. This is what we normally mean when we talk about agents performing a move, rather than simply moving or being moved. Instead of being an equal partner in the mutual coupling with its environment, the system is, at least in some cases, the true source of activity, i.e. in some way responsible for, or the agent of, this activity. Note this does not imply that interactional asymmetry has to be exercised all the time. Some actions might involve active regulation only initially or intermittently. And at times an agent may passively undergo changes that are not actions proper. But also note that in many cases of apparent passivity or automaticity, for instance, when I'm standing still without paying attention to my doing so, or in Dreyfus's examples of driving to work, shuffling to a comfortable distance in the lift, or expert athletic performance, there may still be subpersonal processes of active regulation occurring, such as those involved in balancing to ensure that I won't just fall over, preparing one's body for a rapid return in a tennis match, and so on.

Lastly, when interactions are modulated according to an agent's goals and needs, and thus have a normative dimension - they are actions, as commonly understood. Within the framework of minimal agency, such normativity arises from the self-producing and self-maintaining nature of the precarious system in question. Through its ongoing individuation, the system intrinsically determines what interactions are supportive of its continued existence, and what interactions threaten its survival. In other words, what is good or bad for a minimal agent, is given by its own organization. When the system is sensitive to these norms, and actively modulates it interactions with respect to them, then we speak of the system adaptively regulating its structural coupling with the environment (Di Paolo 2005). Only such regulations, in contrast to other passive happenings, are acts in the strict sense. They originate in the agent, and they can succeed or fail. Intrinsic normativity of this kind is the third requirement for minimal agency.

In summary, a minimal agent is "an autonomous organization capable of adaptively regulating its coupling with the environment according to the norms established by its own viability conditions" (ibid, p. 376). We argue that the nature of agents as selfasserting systems, able to evaluate external affairs in terms of their own viability, is the origin also of a minimal subjectivity. ${ }^{1}$

However, when we talk about human-level agency, much of our everyday behaviour, while taking place within the constraint of biological viability, is underdetermined by it. Many of our actions, for example, acquire intrinsic value "on top" of their organic functionality: movements can be dexterous, postures awkward, a walk elegant, and so on. The question is whether a new form of autonomy and agency may arise at the behavioural level, not fully determined by biological constraints. Following Di Paolo (2005) and Barandiaran (2007), we suggest that the answer is positive, and that the behavioural analogue to biological agency is a network of precarious but interactively self-sustaining sensorimotor schemes, i.e. a self-asserting sensorimotor repertoire, whose adaptive regulation is directed at the preservation of internal coherence and consistency. Could such a system satisfy the three criteria for minimal agency?

\footnotetext{
${ }^{1}$ For further discussion on the enactive notion of autonomy and its relation to sense-making and agency see e.g. Weber and Varela 2002; Di Paolo 2005; Thompson 2007; Di Paolo and Thompson 2014; also Thompson 2011, and critical commentary in the same issue).
} 
Firstly, non-trivial self-assertion of individuality requires that without the system's activity its component processes will cease to exist, in other words that they are precarious. How does this apply to sensorimotor organizations? Firstly, many of our behaviours are habitual in nature. By this we do not mean that they are automatic and stereotypical, but that they (and/or the neurodynamic structures underlying them) are reinforced through their repeated exercise, which they also tend to pre-dispose, and without which they are in danger of being extinguished (Barandiaran and Di Paolo 2014; Carlisle 2014). As is wellknown, skills and habits tend to decay in the absence of frequent enough enactments, hence their precariousness (see Arthur et al. 2013). A self-reinforcing tendency predisposing future enactments can counteract this decay. But excessive robustness also plays against the precariousness requirement. Instead, as part of a greater network, schemes and habits may also depend on other behaviours as preconditions for their exercise. It is precisely this form of co-dependence that furnishes habits or schemes with a certain plastic opening towards novelty. A habit wears grooves and sets limits to itself. But, according to Dewey (1925), this is true of an isolated habit. Interaction between habits "not only increases the number and variety of habits, but tends to link them subtly together, and eventually to subject habit-forming in a particular case to the habit of recognizing that new modes of association will exact a new use of it. Thus habit is formed in view of possible future changes and does not harden so readily", (ibid., p. 281). In this way, the sensorimotor agent is individuated as a complex network of interdependent SM schemes, each helping to sustain the others by avoiding both decay and over-rigidity.

Normativity in a network of sensorimotor structures, we propose, arises from the fact that such a network can be coherent and stable to varying degrees. By this we mean that individual sensorimotor structures may be more or less well adapted to the context in which they are usually enacted and that the SM repertoire may hold SM schemes that are, up to a point, mutually conflicting. It is easy to see how in early developmental stages infants may enact the wrong sensorimotor coordinations (see e.g. the dynamical systems analysis of preservative reaching, the A-not-B error, in infants by Smith and Thelen 2003), or possess conflicting sensorimotor schemes (Piaget 1981, relates the story of children's confusion when first trying to recognise letters that look identical in the mirror and those that do not). Similar perturbations of the repertoire's coherence are to be expected as the agent matures and acquires new skills (and forgets others) throughout her lifetime.

Finally, when the agent constituted by the sensorimotor network becomes sensitive to these norms of coherence and stability, and adaptively guides transformations that conserve its "viability" (interactional asymmetry), we may speak of a system that exhibits agency at the sensorimotor level. Because such systems have an intrinsic concern for their own stability, and evaluate environmental conditions in terms of what is good or bad for themselves, they entail a subjective perspective (Jonas 1966; Thompson 2007), and therefore allow us to speak of them as possessing interiority and enjoying experiences. ${ }^{2}$

\footnotetext{
${ }^{2}$ Sensorimotor identity comprises its own processes of individuation which are different from those that sustain the organic identity of biological agency. The latter constrains sensorimotor agency without fully determining it. We do not discuss here the complex question of how these two forms of agency and identity relate to each other. While conceptually it is important to highlight the distinction between the two kinds of agency, in practice their relation includes the possibility of mutual influence and co-development, leading potentially to integrated personal agency (see also discussion on the social agency in the last section). Habits can shape organic identity, for instance in terms of diet, daily activities, hygiene, exposure to chemicals, pathogens, and so on.
} 
We should clarify at this point that the experiences we propose a sensorimotor agent is able to enjoy differ from the minimal sentience that (mere) organic agency entails. In particular, we claim that sensorimotor agents may experience their actions as their own, and that we can derive the qualities and aspects of how such systems experience their own agency from the way in which they exercise adaptive regulations to maintain a stable sensorimotor repertoire. Piaget has developed exactly such an account of how sensorimotor skills are developed and coherently maintained via adaptive transformations. To this we turn next.

\subsection{SM equilibration}

Piaget's research in the areas of cognitive development and genetic epistemology can be seen as an attempt to determine how higher forms of human understanding (e.g. abstract and rational) arise from earlier and more implicit forms of sensorimotor organization. A fundamental building block in his theory is the concept of a scheme. By this he refers to a kind of behavioural pattern, or action, always required for making (practical) sense of our environment, which matches directly with the level of SMCs we have identified above as "SM schemes". According to Piaget, and in agreement with the sensorimotor approach, the environment is not a set of pre-existing stimulus conditions that impact on the organism to produce a perceptual or cognitive effect. A subject can rather perceive - in the sense of understanding for what it is, or what it is for-only those environmental aspects or events that she can actively assimilate (integrate or absorb) into already existing sensorimotor schemes. The maturing subject, moreover, faced with an ever changing world (and body), will constantly be challenged by not-yet-assimilated aspects of her environment, which create internal sources of tension and conflict in her cognitive organization. Through adaptive processes of accommodation, Piaget proposes, the existing repertoire of sensorimotor schemes is modulated or transformed over time such as to address new behavioural challenges. The subject is thus continuously poised at the edge between assimilation and accommodation, in a process of equilibration through which she reaches new forms of organizational (meta)stability.

Piaget illustrates the stabilization of a SM scheme as the closure of a cycle of more basic sensorimotor coordinations (which again match with the corresponding level of SMCs defined above). So, for instance, a baby's scheme for drinking milk from a bottle (and thus her perception of the bottle as affording such action) may involve the more basic coordinations of latching on, suckling, swallowing, breathing etc., organized appropriately such as to achieve the desired result. Individual coordinations, in turn, depend for their correct exercise on the assimilation of corresponding aspects of the environment, such as the shape of the bottle, the sensation of milk flowing or air being inhaled and so on. Such behavioural patterns can be perturbed in different ways. In Piagetian terms, a subject may experience an obstacle when a previously successful sensorimotor coordination fails to assimilate its corresponding environmental aspect (e.g. failure to latch on to the bottle because the teat's shape has changed). Alternatively, the subject may encounter a lacuna, or gap in the current sensorimotor organization. In this case, something is manifestly unknown about the world since the presumed correct handling of the situation (e.g. latching on) does not lead "as expected" to the next stage in the cycle (e.g. suckling, perhaps because the bottle is empty). 
The process of equilibration reduces the occurrence of such perturbations by modifying or deriving new sensorimotor schemes from existing ones, involving processes such as differentiation, grouping, branching, re-ordering, and so on (resulting, for example, in the creation of a bottle-feeding scheme from a prior breast-feeding scheme). Moreover, processes of equilibration also tend to maintain internal coherence in the repertoire of SM schemes. Piaget suggests, for instance, that reciprocal processes of accommodation and assimilation may also occur between schemes, as well as between schemes and the system's totality (involving a hierarchical dimension of relationships among schemes). Note that the conservation of self-sustaining sensorimotor schemes, and the stability of the SM repertoire as a whole, play the role of adaptive regulations in the definition of minimal agency, and as such may already ground aspects of normativity at the sensorimotor level. What is good or bad for the subject as a sensorimotor agent is, respectively, what can be equilibrated or what provokes tensions or instabilities in his cognitive organization. For an example of how the stability of a SM scheme, rather than some external measure of performance, can guide the learning of a new sensorimotor skill see Kostrubiec et al. (2012).

Piaget's idea of equilibration can be made more explicit by casting it in terms of processes involving SMCs (Di Paolo et al. 2014). Dynamically, there are two conditions for the successful assimilation of an environmental feature or process into a sensorimotor scheme. The stability condition specifies that the pattern or set of environmental states and the pattern or set of the agent's states, when engaged in a sensorimotor coordination, are mutually stabilizing. In other words, the fully coupled agent-environment system, when engaged in such a coordination, produces only trajectories that belong to the same functional class (fulfilling the same role in the task). The transition condition specifies that the production of a stable sensorimotor coordination A leads in time to the production of coordination B, where B is the next stage in the sensorimotor scheme comprising the individual coordinations. On this account, Piaget's lacunae correspond to violations of the transition condition, and obstacles to violations of the stability condition. As we will argue next, these violations may be experienced as breakdowns of agency, and their absence as the feeling of control.

\section{Enactive sense of agency}

We are now in a position to synthesise the three theoretical strands - the dynamical sensorimotor approach, the enactive notion of sensorimotor agency, and the dynamical theory of sensorimotor equilibration - to offer an enactive account for the sense of agency. According to our proposal, a basic experiencing agent is constituted by a selfasserting network of mutually enabling precarious sensorimotor schemes; a network whose stability is constantly challenged by environmental and internal requirements, and which undergoes adaptive processes of equilibration to counter these challenges. Such an organization satisfies the three requirements for minimal agency and may thus be said to constitute an identity that emerges at the sensorimotor level, bearing its own concerns, acting on its own behalf, entertaining its own perspective; in short, a sensorimotor subject. What and how this agent experiences perceptually, according to the sensorimotor approach, is determined by her sensorimotor repertoire. Crucially, we 
propose that how the agent experiences her engagements with the world, i.e., whether as owned and self-driven or not, is determined by different modes of equilibration both within and between SM schemes, and by how these modes of equilibration re-assert the individuation of the sensorimotor subject.

To motivate this idea, consider the following parallel. The sense of agency is generally understood by many as originating in a kind of self-monitoring, i.e. a diffuse awareness of the effect of one's actions on the environment and one's body. This is analogous to the requirement of adaptivity for minimal agency, i.e. an agent's monitoring and adaptive regulation of the consequences of its activities for its own viability. In the context of sensorimotor agency, we have developed Piaget's theory of equilibration as a possible form of adaptivity (see Di Paolo et al. 2014). Our suggestion is that the different aspects of the sense of agency discussed in Section 2 derive from the modes in which a sensorimotor agent monitors and adaptively regulates its interactions such as to stabilise and deploy a precarious network of SM schemes. This will involve both the (meta)stability of SM schemes and the (meta)stability of their mutual relations, i.e., aspects intrinsic to individual actions and aspects concerning how schemes relate to each other.

To be more specific, our proposal states that the different aspects of the sense of agency cohere with the different aspects of assimilation and accommodation in a sensorimotor agent and not with specific relations between sensory signals. For example, we consider the diffuse and attentively recessive feeling of agency to be the experiential consequence of a network of SM schemes successfully assimilating the current environmental context. In other words, the absence of any perturbations to my dynamic equilibrium, is the feeling that "everything is going according to (my) plan". Since SM schemes are always enacted by the sensorimotor agent owning them and their enactment in turn re-constitutes the organization that constitutes the sensorimotor agent, the "first-person givenness" is already implicit in the process that asserts a SM scheme within the precarious network, i.e., its enactment. In other words, whether or not I am the agent of my actions, at this level of equilibration, is not a question of verification based on comparisons between signals, since it always is and can only be I who enacts a SM scheme whose outcome is to contribute to re-affirm my own agency. The enactment of the act asserts the agency of the agent.

The sensorimotor "congruence" required for this pre-reflective feeling of agency is not one involving precise matching of predicted feedback, as in the comparator model. Rather, it is simply the satisfaction of the stability and transition conditions for assimilation (as defined above), in other words, the fact that agent and environment play the roles required for the enactment of the SM scheme to be successful and coherent with the rest of the network. It is thus not a congruence between internal signals in the brain, but an appropriateness of the chosen scheme given the current situation in agent and environment. The agent does not need to "find out" whether a SM scheme equilibrates by comparing signals. It is the enactment of the SM scheme itself that results in success or failure to various degrees (see discussion on intrinsic normativity in SM schemes in Di Paolo, et al. 2014). An obstacle or a lacuna are manifested directly in the failure to equilibrate within an SM scheme or between one SM scheme and another. The manifestation is world-involving and also personal since it implies the agent as a whole. 
While this sense of agency is phenomenologically recessive, we do become more consciously aware of situations where our interactional stability is challenged and an assimilation fails. Such situations correspond to the cases of lacunae and obstacles just mentioned. In the former case, I am, in a sense, successfully engaged in what used to be the correct SM coordination given the context (e.g. I perform the right arm movements to dribble a basketball). But because of changes in the structure of the environment (say, a loose floorboard), or changes in my own body, my enacting of the coordination does not lead to action effects appropriate for achieving the desired outcome usually associated with the full SM scheme; i.e. the transition to the next coordination in the scheme's own organization fails (the ball does not return to my hands, which prevents me from catching it). I will experience this as a lack of agency for the outcomes of my actions. Conversely, in the absence of lacunae, the satisfaction of the transition condition may contribute to the intentional aspect of achievement in my feeling of agency (the feeling of successful dribbling). In the case of obstacles, in contrast, the environment or my body do not "play along", as they usually do in my enactment of a SM coordination (e.g. a deflated ball, or injured arm preventing me from correctly dribbling the ball). What is experienced in this case, is the lack of a sense of control for my action. The basic feeling of agency that derives from aspects of ongoing movement (Table 1), namely the intentional aspect of achieving the desired (distal) effect and the feeling of control (proximal), is thus best described as an absence in our account. It is the feeling that my agency is not currently challenged by lacunae nor obstacles.

Another kind of agency awareness can be associated with features of the ongoing process of active equilibration of SM schemes. Though failure of assimilation may in the first instance raise awareness that I am not in total control of my interactions and their consequences, a second level of awareness may arise from the effort I have to exert in order to re-equilibrate, i.e. to counter perturbations and re-establish a "smooth" interaction. In other words, this sense of agency corresponds to the amount of self-

Table 1 Aspects in the pre-reflective sense of agency and the corresponding processed involved

\begin{tabular}{|c|c|c|}
\hline & Intentional aspects & Movement aspects \\
\hline Initiation & Anticipatory/Preparatory: & Movement specification: \\
\hline $\begin{array}{l}\text { Processes of (attempted) } \\
\text { accommodation. }\end{array}$ & $\begin{array}{l}\text { Awareness of intention prior to } \\
\text { movement. Sense of urge. } \\
\text { Selection between metastable } \\
\text { SM schemes and the covert } \\
\text { effects of the anticipative state } \\
\text { on other parts of the } \\
\text { cognitive organization. }\end{array}$ & $\begin{array}{l}\text { Sense of having initiated one's } \\
\text { movements (early efferent). } \\
\text { Engagement of SM scheme, and } \\
\text { covert effect of a stabilized } \\
\text { committed state on other parts } \\
\text { of the cognitive organization. }\end{array}$ \\
\hline Ongoing/Monitoring & Achievement (task, goal): & Control: \\
\hline $\begin{array}{l}\text { Processes of (attempted) } \\
\text { assimilation. }\end{array}$ & $\begin{array}{l}\text { Sense of achieving the intended } \\
\text { effect (distal feedback about } \\
\text { outcome, action-effect). } \\
\text { Satisfaction of the transition condition. } \\
\text { Lacking or perturbed sense } \\
\text { of achievement in case of lacunae. }\end{array}$ & $\begin{array}{l}\text { Sense of successfully controlling } \\
\text { the current action (afferent } \\
\text {-efferent congruence). } \\
\text { Satisfaction of the stability condition. } \\
\text { Lacking or perturbed sense of } \\
\text { control in case of obstacles. } \\
\text { Effort: } \\
\text { Sense of exerting control. } \\
\text { Adaptive processes of (re-)equilibration. }\end{array}$ \\
\hline
\end{tabular}


correcting activity involved in achieving increased equilibration. As this activity diminishes whenever equilibration approaches its maximum condition, the intensity of the effort-related aspect of agency experience also diminishes. A prediction of this view would be that if we were to perform a very repetitive task, such as a long copy and paste sequence, our sense of effort should be greatest at the beginning, since we have to deploy more regulatory resources to establish a stable interaction to begin with. But as we repeat the actions, and as long as we do not suffer from fatigue, these would come to feel more and more automatic. Once the fluent interaction is established, and not challenged by unexpected events, the initially strong sense of effort is thus replaced by a less intense feeling of being in control (and though the actions may feel "automatic", it never feels as if somebody else is in control).

In this respect, Nahmias (2005) and Pacherie (2007b) acknowledge a certain ambiguity affecting the sense of agency in situations of effortless control. Pacherie suggests introducing a distinction between the feeling of being in control and the feeling of exerting control. The latter is what we may call the kind of agency awareness we associate with progressive equilibration. As equilibration is achieved, the feeling of exerting control becomes ambiguous. Our prediction of a loss of intensity in this feeling during fluent, repetitive tasks (before we reach a situation of fatigue), we note, is quite different from what would be expected from the comparator model, since we cannot introduce the distinction of being in control and exerting control in it. The better the match between predicted and sensed signals, the less the error and therefore the more reaffirmed or confirmed the sense of agency should become, contrary to what we suggest would happen according to our proposal.

Some aspects of agency experience associated with the initiation of movement are identified in Table 1 as the awareness for one's intention and the awareness for having decided to engage (now) in action. We can relate these aspects to the dynamics of how a particular SM scheme in one's repertoire is selected and activated. As sketched above, we envision this process as one of competition between SM structures, whose implementation on the agent-side involve metastable neurodynamic patterns and bodily states, and which resonate with compatible environmental conditions. When the activity of such a SM structure is lifted above a certain level (e.g., through adaptive regulations by the agent), the scheme is activated, coupled to the motor system, and thereby allowed to contribute to overt action (unless movement is prevented). Crucially, the emergence of a preferred scheme (the passage from metastable to more stable neuro- and body dynamics), even if not yet activated at the muscular level, can nevertheless have real consequences for the agent. This corresponds to the agent being in an anticipative state, in which the selection of the scheme may influence other processes in the agent's cognitive organization. The agent's sensitivity to the selected scheme thus constitutes a kind of awareness of processes and events about to ensue, and we identify this sensitivity as coherent with the intentional aspect of action awareness (the awareness of having the intention to move). For example, when a tennis player forms the intention to return his opponent's serve, he is already prepared to move in a certain way (e.g. by setting up required motor primitives and synergies). But at the same he is still sensitive to the different ways the ball may be served, as well as to the different options for his opponent's reaction to his upcoming return. The particular manifold of possibilities associated with the selection of a specific return scheme is experienced by the player as his intention to enact that return. 
The irreversible activation of a scheme, in contrast, implies a commitment to move. Now the agent is less sensitive to the possibilities and tendencies for action that may or may not occur. Instead, his activity is funnelled towards the execution of a single active SM scheme, which again may lead to changes in other parts of the cognitive organization, such as the priming of SM schemes that usually follow the current one. We identify this narrowing down of dynamical possibilities (commitment ${ }^{3}$ ), and the corresponding real consequences for the agent as a whole, as the awareness for action initiation. In our example, the tennis player's engagement of the return action may already have primed his subsequent move towards the net, for instance. The associated collapsing of possible alternative courses of action is experienced by the player as his having initiated the return.

One may also picture the temporal dynamics of the selection and activation of a scheme as the emergence and shaping of a particular basin of attraction in the agent's SM space. The structure of the scheme's basin determines the agent-environment synergies, or constraints on their coupling, that are appropriate in the given task context. This poises the agent in an anticipative state rich in equally valid propensities to respond (this is the intentional aspect). A slight change in circumstances (internal or external) then can be enough to break the system's symmetry and select one amongst many possible actions (the action initiation aspect). This is the same view Wallot and van Orden (2012) defend for explaining cases of so-called ultrafast cognition, i.e., cognitive performances with response times as fast or faster than the estimated physiological limits of information processing. Empirical evidence in their support also counts in favour of our interpretation of SM scheme dynamics. Our crucial addition is that the emergence of a SM scheme's basin of attraction, and the symmetry-breaking, also have covert consequences on other parts of the cognitive organization, which results in the agent's awareness of intention and action initiation respectively.

In general then, the distinction between the sense of being the initiator of an action (associated with intentions and premotor activity), and the sense of being in control (on-going motor-sensory matching) can be neurophenomenologically matched to processes of accommodation and assimilation respectively. Note, that in terms of equilibration, the initiation of a new SM scheme is already an adaptive regulation, i.e. a response to a new external or internal challenge to stability. The feeling of being the initiator of an action would thus not be limited only to cases of prior losses of control over one's actions.

\footnotetext{
${ }^{3}$ The notion of commitment, seemingly vague, has been measured in simulation models of minimal cognition and indirectly in neuroscientific studies. When a simulated agent must make a choice between alternative behaviours, one can systematically study the effect of variations in the agent's or environmental states on the final choice made by the agent. One can explore this effect over time and find counter-intuitively that commitment to a choice is not a monotonic quantity that increases progressively as the time for execution approaches but that instead it can fluctuate in complex ways (Beer 2003). Alternatively, one can 'freeze' time just before the simulated agent has made its choice and ask the question 'what if things had been slightly different?' and then quantify the effect of changes to the environment on the decision - the larger the effect, the less the commitment to the action that is nevertheless actualized (Iizuka and Di Paolo 2007; Di Paolo and Iizuka 2008). In the case of humans the stability of neural cortical patterns may be measured indirectly in single perceptual trials by analyzing the statistical variability corresponding to different cortical regions. Evidence shows that low variability or other measures of coherence between recorded patterns indicating a more stable global state correspond to the emergence of a commitment to a conscious perceptual choice (Schurger et al. 2015; Varela et al. 2001).
} 
Different aspects in the sense of agency may further be mapped to the type of assimilation condition being challenged or the different forms of equilibration (summarised in Table 1). As we have said, intentional aspects of action awareness may be related more with the occurrence of lacunae, and movement-related aspects more with obstacles. Without being able to develop a full story here, one may also relate the distinction by Marcel (2003) between short and long-term senses of agency to the different kinds of equilibration. For instance, equilibration occurring to address incongruence between a SM scheme and the environment here and now may underlie the instantaneous feeling of agency. In contrast, adaptive processes aiming to resolve mutual conflicts between SM schemes, or between a SM scheme and the repertoire as a whole, may underlie the general feeling of being reliably effective in one's actions, and of possessing the necessary sensorimotor skills to deal with one's everyday life. In this sense, the higher forms of equilibration may be necessary for the experience of oneself as a unified, persisting and coherent source of intentional activity over time.

As an example of the applicability of our proposal consider passivity experience in schizophrenic patients with delusions of control. These patients do not disown the intentional aspect of their actions. They successfully follow instructions by an experimenter, and do not try to stop the affected movements. They report, however, not to have been responsible for initiating their actions, i.e. not to have consciously intervened in their execution (passivity). In other words, they have correct awareness of their intentions, but not of action initiation.

According to the comparator hypothesis, this aspect of agency experience results primarily from efferent signals, i.e., from the flow of motor specifications that are responsible for translating intentions into movement and occur prior to their execution. On one account (Frith et al. 2000a), it is suggested that the passivity experience arises from an impaired predictor of limb position. While the patient is aware of his intentions, and while these are translated into the correct movements and achieve the desired effect, in the absence of predictions at the comparator (both $C_{1}$ and $C_{2}$ in Fig. 1), the patient is not aware of the precise specification for movement, and as a result, it is presumed, of his initiating it. The situation at the comparator is as if the movement was passive, and the afferent feedback is thus attributed to external causes rather than to the self.

From our enactive perspective there is no need for central predictors to account for the awareness of action initiation. ${ }^{4}$ As we have argued, awareness of intention and action initiation results from the real but covert effects that the selection and activation of SM schemes have on the rest of the agent's cognitive organization. In particular, the activation of a specific SM scheme places the agent in a state of "commitment" that may constrain future actions by differentially modulating other SM schemes that usually follow. Awareness of action initiation may be disrupted, resulting in passivity experience, when a SM scheme's activation no longer has the right secondary effects on the remaining SM repertoire, i.e. when the scheme is executed as if decoupled from the greater network. This view is compatible, for example, with speculations by Frith et al. (2000a) regarding the underlying neurological abnormalities in patients with delusions

\footnotetext{
${ }^{4}$ That is not to say that sensorimotor behaviours or schemes cannot be anticipative in nature, but rather that each scheme, if required, may be intrinsically anticipative rather than relying necessarily on detailed internal models (see Stepp and Turvey 2010).
} 
of control. The authors argue that the overactivity in the inferior parietal cortex, as observed in some patients, may be due to abnormal modulation via the anterior cingulate, which in turn is speculated to be involved in attention to future actions and suppression of inappropriate actions.

In short, abnormal awareness of action initiation may be due to impaired coupling between different SM schemes, which in normal circumstances results in anticipative modulation of future actions. Such an impairment would not interfere with the actual execution of a SM scheme, which is compatible with the fact that schizophrenic patients have no problem with motor control itself. A prediction may be, however, that rapid execution of (unusual, non-automatic) action sequences could be impaired, since the selection of one scheme does not always correctly prime other relevant ones ahead of time. Though we are not aware of any experiments testing this specifically, there is evidence that schizophrenic patients, though not having problems with anticipative motor control per se, show indeed a deficit in the sequencing of motor actions (Delevoye-Turrell et al. 2003).

\section{Discussion}

Our hypothesis fulfils several requirements that we believe should apply to any enactive account of the sense of agency. Firstly, the experience of agency, like any other experience in the enactive approach, is relational in nature, i.e. fundamentally world-involving, rather than internal to the brain. It is constituted by structures or processes present in our active exploration of the world, by properties or modes of the relation between agent and environment.

Secondly, the prereflective sense of agency, in our account, is an intransitive experience. It is not an experience of 'me' as an object of perception (as defended e.g. by Bayne 2011) or introspection, but rather the basic feeling of my intentional directedness at the world; i.e. the feeling of (re-)asserting myself as an agent in meaningful interactions with the world. Thus, our proposal does not reduce the sense of agency to an epistemic issue (accessing information to verify authorship) but also accounts for the ontological question of how a SM scheme participates in the ongoing constitution of the agent; the epistemic issue comes out in the wash.

Thirdly, evidence provided for the comparator model suggests that some form of congruence between different motor and sensory streams is, or can be, involved in the feeling of agency. Our hypothesis accounts for this congruence (satisfaction of assimilation conditions), and its breakdown (lacunae and obstacles), in a more general way than matching signals and without requiring precise predictions or internal representations.

Fourthly, our proposal accounts for the fact that the sense of agency phenomenologically presents itself as a heterogeneous collection of different ways or aspects of feeling in control that depends on context, the task, the person's history and capacities, and so on. Since individual SM schemes are by definition task-specific, and therefore vary for example in terms of the sensory modalities involved, the balance of contributions from agent-internal and environmental processes etc., it follows that the sense of agency should vary from situation to situation and, when it breaks down, it does so in accordance with the specific demands and properties of the task and person. 
Fifthly, our proposal coheres naturally with the phenomenological recessiveness of the feeling of agency as the absence of perturbations or instabilities in an agent's active assimilation of its environment. Conversely, the fact that we often become aware only of our loss of agency, when our intentions are thwarted or our sensorimotor engagements unexpectedly disrupted, is explained by the occurrence of violations of the assimilation conditions.

Lastly, by satisfying the three requirements for minimal agency, our proposal solves the problem of who is experiencing by positing that there exists a well-defined subject that is experiencing its own agency, namely a sensorimotor agent constituted by a selfsustaining network of precarious sensorimotor schemes. Such an agent is invested in interactions with its own intrinsic norms, and its very constitution brings forth a domain of self-relevant interactions, and therewith an intrinsic subjectivity and perspective on the world (Di Paolo 2005; Jonas 1966; Thompson 2007). In this sense, our account spans both the subpersonal level of sensorimotor processes, as well as the personal level of the experiencing subject, something the comparator model fails to do satisfactorily.

An additional aspect that distinguishes our proposal from most accounts of the sense of agency (e.g. Marcel 2003; Synofzik et al. 2008; Pacherie 2007b), is that it conceives of actions and intentions as inseparable qualitative aspects of sense-making (in the sense of Merleau-Ponty's motor intentionality, 2012/1945; also see Gallagher and Miyahara 2012). In our view, all actions are by definition intentional; equally there is no such thing as an abstract intentional 'state' as divorced from the action that it requires for its realization, even if for whatever reason such action is not fully actualised. The intentional aspect of an action derives from the dispositions that the agent exhibits when a SM scheme is selected from the greater repertoire; and from the fact that the selection itself involves the agent's needs and desires. For a variety of reasons, however, an action may not be (fully) realized. In this case the intentional aspect may manifest itself without any overt movement (Di Paolo 2015). This manifestation may nevertheless have real consequences for the agent, and as such may underlie the experience of agency (as well as perceptual experience), in situations that do not seem to involve movement or the world, such as illusions, hallucinations, or in case of paralysis or locked-in syndrome (Kyselo and Di Paolo, 2014, see also Beaton 2013).

Our proposal has assumed a certain phenomenology of agency experience as unquestionable, in particular the distinction between a prereflective feeling and a conceptual judgement of agency. However, this phenomenological account is by no means universally accepted. Indeed, it is not uncommon to question whether there is any distinctive feeling of agency at all, or at least its status as a distinct kind of experience. We cannot here respond in detail to all existing opinions about the epistemological and ontological status of agency experiences. We limit ourselves to highlight a few alternatives and their relation to our proposal.

On one line of thought, for example, the sense of agency is presumed to derive from retrospective conceptual inference about one's intentions and observed events (Stephens and Graham 2000; Wegner and Wheatley 1999; Alsmith 2015). For proponents of this doxastic model the "sense" of agency is not a type of experience at all, but rather a species of belief. We agree with Bayne (2011) in that everyday intuition, the results of experimental manipulation, and observations from pathological cases provide sufficient evidence for the separability between the experience of agency and conscious judgements or cognitive inferences about it. 
Equally extreme is Dreyfus's account of skillful absorbed coping (2007), in which he claims that in such situations not only is there a loss of conscious awareness of - and reflection on - what one is doing, but also a complete loss of any non-reflective experience whatsoever: "In general, when one is totally absorbed in one's activity, one ceases to be a subject" (ibid., p. 373). All there is, on this account, are automatic happenings in response to dispositional sensitivities that have been tuned in the process of mastery of the relevant skill. "In fully absorbed coping, there is no immersed ego, not even an implicit one. The coper [...] only needs to be capable of entering a monitoring stance if the brain [...] sends an alarm signal that something is going wrong" (ibid, p. 374). We agree with Zahavi (2013) here, in that Dreyfus seems to be going too far in rejecting the role of subjective awareness in absorbed coping by implying that there can only be conscious monitoring or no subjectivity at all, while not considering the possibility of an implicit first-personal givenness in experience (as well as subpersonal monitoring and regulation, which we believe would still be occurring in the highly skilled, if absorbed, activity he refers to). As Zahavi notes, at least in this point Dreyfus seems to be at odds with the phenomenological tradition with which he associates himself. This first-personal givenness, in our view, partly manifests itself in the phenomenological recessiveness of the sense of agency during skilful coping. This, as we have said, may best be described as a feeling of absence (of friction or challenges impeding my coping). Importantly, a feeling of absence is not the same as the absence of a feeling.

Defenders of the thetic model, in contrast, while not denying the experience of agency as such, claim that the sense of agency is but another form of perception, namely a transitive experience of certain things being the case about one's intentional actions; in other words, involving representations of certain facts about my actions that are available to my awareness. Such states are understood to have a mind to world direction, in so far as they are verified as true when they fit the world, and otherwise non-veridical (also see Bayne 2011, for a more detailed discussion of this school of thought; as well as De Vignemont 2013, and Bermúdez 2015, for a related discussion in the context of sense of ownership). The comparator model squarely falls into this category, which we reject for reasons already given in sections 3 and 4.2.

Another example of the thetic approach is Grünbaum's (2015) argument that what subjects report (or experimenters interpret) as a feeling of agency, may ultimately be reducible to the awareness for one's conscious intentions (possibly based on premotor processes involving intentions, planning and action-selection), and therefore not a distinct kind of agency experience after all. In this respect we note that by considering only the case of an "intention-free" feeling of agency (ibid., p. 21), the author implicitly rules out the possibility that the experience of agency may be distinct in virtue not of its irreducibility to conscious intentions, but of its being implicit in the first-personal givenness or egocentricity of (motor-)intentional actions; which we have here spelled out as the reaffirmation of a sensorimotor system's agency in intentional action. Elsewhere the author argues that we have the "experience of acting intentionally" when non-propositional practical sensorimotor anticipations are fulfilled sensorily (Grünbaum 2011). This seems to match closely our account of successfully assimilating sensorimotor coordinations, and in our view this is one aspect of the kind of intransitive agency experience the author aims to deny. The difference seems to be i) that these anticipations, according to Grünbaum, are derived subpersonally from basic 
propositional intentions, and ii) that it is the role of the agentive experience to justify the agent's belief about his actions ("which is formed by grasping the content of one's basic intention as a description of one's action"). We believe that our enactive proposal shows how in everyday skillful behavior, the sense of agency does not (only) play this epistemological role, but is rather an intrinsic aspect of how meaningful sensorimotor schemes are organised and enacted in the world. Propositional beliefs need not be involved at all according to our proposal.

The view we defend in this paper is best described as a telic model: the sense of agency is understood as an intransitive experience that is implicit in intentional action itself, i.e. such actions are considered to be enjoying experiential character, or phenomenal properties themselves (see, e.g., Searle 1983). Telic states have a world to mind direction of fit. Their aim is to bring about certain changes in the world. They are satisfied when an intentional action is successfully realized (assimilation), or otherwise fail and remain unsatisfied or frustrated (requiring accommodation). According to our proposal, the prereflective feeling of agency is of this kind. It is tightly linked with the reaffirmation of an agent as an agentive system through its actions. As such, I cannot have a feeling of agency for a movement if I am not the agent of it, i.e. this feeling cannot be non-veridical (though my reflective judgement could). Regarding support for such a non-observational approach to action awareness also see Marcel (2003).

Since ours is a meso-level proposal, we have not said much about how SM scheme equilibration and selection is supported in the brain and other body structures, other than that we envision the involvement of metastable neuro(body)dynamic patterns. SM schemes recruit structures distributed across many brain and body regions, involving different sensory modalities, motor control areas etc. Premotor areas and what are usually considered higher-level cognitive areas, such as prefrontal cortex, are expected to contribute to the selection and mutual priming of SM schemes. Thus, the fact that we find evidence for neural processes in support of the comparator model (see e.g. David et al. 2008, for a review) does not immediately contradict our proposal. The different stages of scheme equilibration, selection, and activation may well involve activity in similar brain areas as those associated with stages of the comparator model. We have presented our proposal in contrast with the comparator model, which we find neither necessary nor sufficient for explaining the sense of agency. But we do not claim that similar neural processes may not support SM schemes and their equilibration. It may well be that a scheme's satisfying of the stability condition, for example, may be implemented through a comparison of different neural signal streams. But such a comparison would only plays an instrumental role in the more fundamental dynamics of SM scheme equilibration, which could also occur through different processes.

An important element that we have not addressed in this paper concerns the social aspects of the sense of agency as well as the constitution of social forms of agency. While the picture given in this paper is altered in such cases, it changes following along the same enactive principles, i.e., we do not need to invoke representational explanations or higher forms of inference or simulation (e.g., Leslie et al. 2004; Goldman 2006; Apperly 2010).

It has been amply demonstrated that in situations of social interaction people extensively coordinate with each other at various levels, from physiological variables, posture, distance, gestures, speech acts, and affect (see e.g. Streeck et al. 2011; Tschacher et al. 2014; Konvalinka et al. 2011; Abney et al. 2014). These coordination 
patterns are part of how individual SM schemes are enacted. Social agency is characterized by what McGann and De Jaegher (2009) call "self-other contingencies". Action and perception in the social domain are a matter of coordinating the behaviours, emotions, and intentions of the agents involved, in and through the coordination of movement (including utterances). Self-other contingencies are different from SMCs in a number of ways. Social interactions are interactions between agents, each of whom is maintaining their own autonomy. The condition of asymmetry between agent and environment is more complex (able to change over time along different dimensions), since the regulation of social interactions is not completely down to either individual (De Jaegher and Froese 2009). As a consequence, interactions with other social agents are far less predictable than those with (most) objects. In this way, the equilibration of SM schemes in interactive situations may not obviously arise from a single agent but could in principle be co-authored, leading to ambiguities, for instance, like controlling one's actions without exerting control (like we described above for the case of repetitive tasks).

Social forms of agency can also be manifested in solitude. In their enactive approach to language, Cuffari et al. (2015) distinguish increasingly complex forms of social agency, from basic coordination in interaction, to partially complementary social acts (e.g., the act of giving/accepting), to acts of mutual control, mutual and self-interpretation, and social self-control (the application of social regulatory acts to the self, as in behaviour directed by inner speech). Some of these socially constituted forms of agency can be manifested in the form of top-down impositions of control and norms. The reflective sense of agency we have mentioned at the beginning of this paper, which involves an introspective stance and the explicit conceptual attribution of agency to myself, as well as narrative skills in some cases, may derive from counterparts of social skills such as self-interpretation and social self-control described by Cuffari et al. A full account of the sense of agency in human beings will have to distinguish the biological, the sensorimotor, and the social forms of agency but also combine them through a core explanatory continuity.

\section{Conclusion}

We have interpreted the sensorimotor approach as a world-involving perspective on action and perception. Lacking a theory of agency, this approach needed to be supplemented by extending the requirements for agency proposed by enactive theory - individuality, asymmetry, and normativity - to a new enactive concept of sensorimotor agency. We have combined this concept with recent formalizations of the notions of sensorimotor contingencies and their mastery. The latter, based on a dynamical account of equilibration, furnishes the enactive approach with a proposal for explaining the sense of agency. A world-involving, non-representational, meso-level account based on how actions and dispositions are organized as a network of precarious, mutually stabilizing sensorimotor schemes. A given act contributes to the ongoing regeneration of this organization to different degrees or fails to do so. It is the self-asserting logic of this network that determines whether an act belongs to the agent or not. Conversely, it is the ways in which an agent acts in the world that individuate her as the agent she is constantly becoming. 
This meaningful relation between agent and world that can be established, lost, and regained, is what in our view best coheres with the phenomenology of the sense of agency.

Acknowledgments The authors are grateful to the reviewers and to Marek McGann and Fernando Bermejo for their comments and recommendations on an earlier version of this article. This work is funded by a Basque Government grant for research groups (IT 590-3).

Open Access This article is distributed under the terms of the Creative Commons Attribution 4.0 International License (http://creativecommons.org/licenses/by/4.0/), which permits unrestricted use, distribution, and reproduction in any medium, provided you give appropriate credit to the original author(s) and the source, provide a link to the Creative Commons license, and indicate if changes were made.

\section{References}

Abney, D. H., Paxton, A., Dale, R., \& Kello, C. T. (2014). Complexity matching in dyadic conversation. Journal of Experimental Psychology General, 143(6), 2304-2315. doi:10.1037/xge0000021.

Alsmith, A. J. T. (2015). Mental activity and the sense of ownership. Review of Philosophy and Psychology, 116. 10.1007/s13164-014-0208-1.

Apperly, I. (2010). Mindreaders: The cognitive basis of "theory of mind.". Hove, East Sussex: Psychology Press.

Arthur, W., Jr., Day, E. A., Bennett, W., Jr., \& Portrey, A. M. (Eds.). (2013). Individual and team skill decay: The science and implications for practice. New York, NY: Routledge.

Barandiaran, X. E. (2007). Mental Life: conceptual models and synthetic methodologies for a post-cognitivist psychology. In B. Wallace, A. Ross, J. Davies, \& T. Anderson (Eds.), The World, the Mind and the Body: Psychology after cognitivism (pp. 49-90). Imprint Academic.

Barandiaran, X. E., \& Di Paolo, E. (2014). A genealogical map of the concept of habit. Frontiers in Human Neuroscience, 8, 522. doi:10.3389/fnhum.2014.00522.

Barandiaran, X., Di Paolo, E., \& Rohde, M. (2009). Defining agency:individuality, normativity, asymmetry and spatio-temporality in action. Adaptive Behavior, 17(4), 1-13.

Bayne, T. (2011). The Sense of Agency. In F. Macpherson (Ed.), The Senses: Classic and Contemporary Philosophical Perspectives.

Beaton, M. (2013). Phenomenology and embodied action. Constructivist Foundations, 8(3), 298-313.

Beer, R. D. (2003). The dynamics of active categorical perception in an evolved model agent. Adaptive Behavior, 11(4), 209-243. doi:10.1177/1059712303114001.

Bermúdez, J. L. (2015). Bodily ownership, bodily awareness and knowledge without observation. Analysis (2015), 75(1), 37-45. doi:10.1093/analys/anu119.

Buhrmann, T., Di Paolo, E., \& Barandiaran, X. E. (2013). A dynamical systems account of sensorimotor contingencies. Frontiers in Cognition, 4, 285. doi:10.3389/fpsyg.2013.00285.

Carlisle, C. (2014). On habit. New York: Routledge.

Christoff, K., Cosmelli, D., Legrand, D., \& Thompson, E. (2011). Specifying the self for cognitive neuroscience. Trends in Cognitive Sciences, 15(3), 104-112.

Clark, A. (2013). Whatever next? Predictive brains, situated agents, and the future of cognitive science. The Behavioral and Brain Sciences, 36(3), 181-204. doi:10.1017/S0140525X12000477.

Cuffari, E. C., Di Paolo, E., \& De Jaegher, H. (2015). From participatory sense-making to language: There and back again. Phenomenology and the Cognitive Sciences. doi:10.1007/s11097-014-9404-9.

David, N., Newen, A., \& Vogeley, K. (2008). The "sense of agency" and its underlying cognitive and neural mechanisms. Consciousness and Cognition, 17(2), 523-534. doi:10.1016/j.concog.2008.03.004.

De Jaegher, H., \& Froese, T. (2009). On the role of social interaction in individual agency. Adaptive Behavior, 17(5), 444 -460. doi:10.1177/1059712309343822.

De Vignemont, F. (2013). The mark of bodily ownership. Analysis, 73(4), 643-651. doi:10.1093/analys/ ant080.

De Vignemont, F., \& Fourneret, P. (2004). The sense of agency: a philosophical and empirical review of the "Who" system. Consciousness and Cognition, 13(1), 1-19. doi:10.1016/S1053-8100(03)00022-9. 
Delevoye-Turrell, Y., Giersch, A., \& Danion, J.-M. (2003). Abnormal sequencing of motor actions in patients with schizophrenia: evidence from grip force adjustments during object manipulation. The American Journal of Psychiatry, 160(1), 134-141.

Dewey, J. (1925). Experience and nature. Chicago, IL: Open Court.

Di Paolo, E. (2005). Autopoiesis, adaptivity, teleology, agency. Phenomenology and the Cognitive Sciences, 4(4), 429-452. doi:10.1007/s11097-005-9002-y.

Di Paolo, E. (2015). Interactive time-travel: on the intersubjective retro-modulation of intentions. Journal of Consciousness Studies, 22(1-2), 49-74.

Di Paolo, E., \& Iizuka, H. (2008). How (not) to model autonomous behaviour. Biosystems, 91(2), 409-423. doi:10.1016/j.biosystems.2007.05.016.

Di Paolo, E., \& Thompson, E. (2014). The enactive approach. In L. Shapiro (Ed.), The routledge handbook of embodied cognition (pp. 68-78). Oxford: Routledge Press.

Di Paolo, E., Barandiaran, X. E., Beaton, M., \& Buhrmann, T. (2014). Learning to perceive in the sensorimotor approach: Piaget's theory of equilibration interpreted dynamically. Frontiers in Human Neuroscience, 8, 551. doi:10.3389/fnhum.2014.00551.

Dretske, F. (2003). Experience as representation. Philosophical Issues, 13(1), 67-82. doi:10.1111/1533-6077. 00005.

Dreyfus, H. L. (2007). Response to McDowell. Inquiry: An Interdisciplinary Journal of Philosophy, 50(4), 371-377. doi:10.1080/00201740701489401.

Engel, A. K., Maye, A., Kurthen, M., \& König, P. (2013). Where's the action? The pragmatic turn in cognitive science. Trends in Cognitive Sciences, 17(5), 202-209. doi:10.1016/j.tics.2013.03.006.

Erlhagen, W., \& Schöner, G. (2002). Dynamic field theory of movement preparation. Psychological Review, $109(3), 545-572$.

Friston, K. (2010). The free-energy principle: a unified brain theory? Nature Reviews Neuroscience, 11(2), 127-138. doi:10.1038/nrn2787.

Frith, C. (2005). The self in action: lessons from delusions of control. Consciousness and Cognition, 14(4), 752-770. doi:10.1016/j.concog.2005.04.002.

Frith, C. D., Blakemore, S. J., \& Wolpert, D. M. (2000a). Abnormalities in the awareness and control of action. Philosophical transactions of the royal society of London. Series B, Biological Sciences, 355(1404), 1771-1788. doi:10.1098/rstb.2000.0734.

Frith, C. D., Blakemore, S., \& Wolpert, D. M. (2000b). Explaining the symptoms of schizophrenia: abnormalities in the awareness of action. Brain research. Brain Research Reviews, 31(2-3), 357-363.

Fuchs, T. (2011). The brain-a mediating organ. Journal of Consciousness Studies, 18(7-8), 7-8.

Gallagher, S. (1997). Mutual enlightenment: recent phenomenology in cognitive science. Journal of Consciousness Studies, 4(3), 195-214.

Gallagher, S. (2000). Philosophical conceptions of the self: implications for cognitive science. Trends in Cognitive Sciences, 4(1), 14-21.

Gallagher, S. (2007). The natural philosophy of agency. Philosophy Compass, 2(2), 347-357.

Gallagher, S. (2012). Multiple aspects in the sense of agency. New Ideas in Psychology, 30(1), 15-31. doi:10. 1016/j.newideapsych.2010.03.003.

Gallagher, S., \& Miyahara, K. (2012). Neo-Pragmatism and Enactive Intentionality. In J. Schulkin (Ed.), New Directions in Philosophy and Cognitive Science: Adaptation and Cephalic Expression. Palgrave Macmillan.

Goldman, A. (2006). Simulating Minds: The Philosophy, Psychology, and Neuroscience of Mindreading (Vol. 144). Oxford University Press.

Grünbaum, T. (2011). Perception and non-inferential knowledge of action. Philosophical Explorations: An International Journal for the Philosophy of Mind and Action, 14(2), 153-167. doi:10.1080/13869795. 2011.569746.

Grünbaum, T. (2015). The feeling of agency hypothesis: a critique. Synthese. doi:10.1007/s11229-015-0704-6.

Grush, R. (2004). The emulation theory of representation: motor control, imagery, and perception. Behavioral and Brain Sciences, 27(03), 377-396.

Holst, E., \& Mittelstaedt, H. (1950). Das Reafferenzprinzip. Naturwissenschaften, 37(20), 464 476. doi:10. 1007/BF00622503.

Iizuka, H., \& Di Paolo, E. (2007). Toward Spinozist robotics: exploring the minimal dynamics of behavioral preference. Adaptive Behavior, 15(4), 359-376. doi:10.1177/1059712307084687.

Jonas, H. (1966). The phenomenon of life: Toward a philosophical biology. New York: Harper \& Row.

Knill, D. C., \& Pouget, A. (2004). The Bayesian brain: the role of uncertainty in neural coding and computation. Trends in Neuroscience, 27(12), 712-719. 
Konvalinka, I., Xygalatas, D., Bulbulia, J., Schjødt, U., Jegindø, E-M., Wallot, S., Van Orden, G., \& Roepstorff, A. (2011). Synchronized arousal between performers and related spectators in a firewalking ritual. Proceedings of the National Academy of Sciences, 108, 8514-8519. doi:10.1073/pnas. 1016955108.

Kostrubiec, V., Zanone, P.-G., Fuchs, A., \& Kelso, J. A. S. (2012). Beyond the blank slate: routes to learning new coordination patterns depend on the intrinsic dynamics of the learner-experimental evidence and theoretical model. Frontiers in Human Neuroscience, 6, 222. doi:10.3389/fnhum.2012.00222.

Leslie, A. M., Friedman, O., \& German, T. P. (2004). Core mechanisms in "theory of mind.". Trends in Cognitive Sciences, 8(12), 528-533. doi:10.1016/j.tics.2004.10.001.

Lutz, A., \& Thompson, E. (2003). Neurophenomenology - integrating subjective experience and brain dynamics in the neuroscience of consciousness. Journal of Consciousness Studies, 10(9-10), 31-52.

Marcel, A. J. (2003). The sense of agency: Awareness and ownership of action. In J. Roessler \& N. Eilan (Eds.), Agency and self-awareness: Issues in philosophy and psychology (pp. 48-93). Oxford: Clarendon.

Marchetti, C., \& Della Sala, S. (1998). Disentangling the alien and anarchic hand. Cognitive Neuropsychiatry, 3(3), 191-207. doi:10.1080/135468098396143.

Margulis, L. (2001). The conscious cell. Annals of the New York Academy of Sciences, 929, 55-70.

McGann, M., \& De Jaegher, H. (2009). Self-other contingencies: enacting social perception. Phenomenology and the Cognitive Sciences, 8(4), 417-437. doi:10.1007/s11097-009-9141-7.

McGann, M., De Jaegher, H., \& Di Paolo, E. (2013). Enaction and psychology. Review of General Psychology, 17(2), 203-209. doi:10.1037/a0032935.

Merleau-Ponty, M. (2012). Phenomenology of Perception. (D. Landes, Trans.) (2nd ed.). London: Routledge.

Metzinger, T. (2000). The Subjectivity of Subjective Experience: A Representationist Analysis of the FirstPerson Perspective. In T. Metzinger (Ed.), Neural Correlates of Consciousness (pp. 285-306). MIT Press.

Nahmias, E. (2005). Agency, authorship, and illusion. Consciousness and Cognition, 14(4), 771-785. doi:10. 1016/j.concog.2005.07.002.

Noë, A. (2004). Action in perception. MIT Press.

O'Regan, J., \& Noë, A. (2001). A sensorimotor account of vision and visual consciousness. Behavioral and Brain Sciences, 24, 939-1031.

Pacherie, E. (2007a). The anarchic hand syndrome and utilization behavior: a window onto agentive selfawareness. Functional Neurology, 22(4), 211-217.

Pacherie, E. (2007b). The sense of control and the sense of agency. Psyche, 13(1), 1-30.

Piaget, J. (1981). Experiments in contradiction. Chicago: Univ of Chicago Pr.

Ramachandran, V. S., \& Rogers-Ramachandran, D. (1996). Synaesthesia in phantom limbs induced with mirrors. Proceedings. Biological Sciences / The Royal Society, 263(1369), 377-386. doi:10.1098/rspb. 1996.0058

Schöner, G., \& Dineva, E. (2007). Dynamic instabilities as mechanisms for emergence. Developmental Science, 10(1), 69-74. doi:10.1111/j.1467-7687.2007.00566.x.

Schurger, A., Sarigiannidis, I., Naccache, L., Sitt, J. D., \& Dehaene, S. (2015). Cortical activity is more stable when sensory stimuli are consciously perceived. Proceedings of the National Academy of Sciences, 112(16), E2083-E2092. doi:10.1073/pnas.1418730112.

Searle, J. (1983). Intentionality. Cambridge: Cambridge University Press.

Seth, A. K. (2014). A predictive processing theory of sensorimotor contingencies: explaining the puzzle of perceptual presence and its absence in synesthesia. Cognitive Neuroscience, 5(2), 97-118. doi:10.1080/ 17588928.2013 .877880 .

Sheets-Johnstone, M. (1999). Emotion and movement. A beginning empirical-phenomenological analysis of their relationship. Journal of Consciousness Studies, 6(11-12), 259-277.

Smith, L. B., \& Thelen, E. (2003). Development as a dynamic system. Trends in Cognitive Sciences, 7(8), 343-348. doi:10.1016/S1364-6613(03)00156-6.

Steinbuch, J. G. (2012). Beytrag Zur Physiologie Der Sinne. Nabu Press.

Stephens, G. L., \& Graham, G. (2000). When self-consciousness breaks: Alien voices and inserted thoughts. Cambridge: The MIT Press.

Stepp, N., \& Turvey, M. T. (2010). On strong anticipation. Cognitive Systems Research, 11(2), 148-164. doi: 10.1016/j.cogsys.2009.03.003.

Streeck, J., Goodwin, C., \& LeBaron, C. D. (Eds.). (2011). Embodied interaction: Language and body in the material world. New York: Cambridge University Press.

Synofzik, M., Vosgerau, G., \& Newen, A. (2007). Beyond the comparator model: a multifactorial two-step account of agency. Consciousness and Cognition, 17(1), 219-239. doi:10.1016/j.concog.2007.03.010. 
Synofzik, M., Vosgerau, G., \& Newen, A. (2008). I move, therefore I am: a new theoretical framework to investigate agency and ownership. Consciousness and Cognition, 17(2), 411-424. doi:10.1016/j.concog. 2008.03.008.

Thompson, E. (2005). Sensorimotor subjectivity and the enactive approach to experience. Phenomenology and the Cognitive Sciences, 4(4), 407-427.

Thompson, E. (2007). Mind in Life: Biology, Phenomenology and the Sciences of Mind. Harvard University Press.

Thompson, E. (2011). Précis of mind in life. Journal of Consciousness Studies, 18, 10-22.

Tognoli, E., \& Kelso, J. A. S. (2014). The metastable brain. Neuron, 81(1), 35-48. doi:10.1016/j.neuron.2013. 12.022 .

Tsakiris, M., \& Haggard, P. (2005). Experimenting with the acting self. Cognitive Neuropsychology, 22(3-4), 387-407. doi:10.1080/02643290442000158.

Tschacher, W., Rees, G. M., \& Ramseyer, F. (2014). Nonverbal synchrony and affect in dyadic interactions. Personality and Social Psychology, 5, 1323. doi:10.3389/fpsyg.2014.01323.

Varela, F. (1995). Neurophenomenology: a methodological remedy for the hard problem. Journal of Consciousness Studies, 3(4), 330-349.

Varela, F. (1999). The Specious Present: A Neurophenomenology of Time Consciousness. In J. Petitot, F. J. Varela, B. Pacoud, \& J.-M. Roy (Eds.), Naturalizing Phenomenology (pp. 266-314). Stanford University Press.

Varela, F. J., Thompson, E., \& Rosch, E. (1991). The embodied mind: Cognitive science and human experience. Cambridge, Mass: MIT Press.

Varela, F., Lachaux, J.-P., Rodriguez, E., \& Martinerie, J. (2001). The brainweb: phase synchronization and large-scale integration. Nature Reviews Neuroscience, 2(4), 229-239. doi:10.1038/35067550.

Wallot, S., \& Van Orden, G. (2012). Ultrafast cognition. Journal of Consciousness Studies, 19(5-6), 141-160.

Weber, A., \& Varela, F. J. (2002). Life after Kant: natural purposes and the autopoietic foundations of biological individuality. Phenomenology and the Cognitive Sciences, 1(2), 97-125.

Wegner, D. M., \& Sparrow, B. (2004). Authorship Processing. In The cognitive neurosciences (3rd ed.) (pp. 1201-1209). Cambridge, MA, US: MIT Press.

Wegner, D. M., \& Wheatley, T. (1999). Apparent mental causation: sources of the experience of will. American Psychologist, 54, 480-492.

Wolpert, D. M., Doya, K., \& Kawato, M. (2003). A unifying computational framework for motor control and social interaction. Philosophical Transactions of the Royal Society of London. Series B, Biological Sciences, 358(1431), 593-602. doi:10.1098/rstb.2002.1238.

Zahavi, D. (2013). Mindedness, mindlessness and first-person authority. In J. Schear (Ed.), Mind, reason and being-in-the-world: The McDowell-Dreyfus debate (pp. 320-343). London: Routledge. 